\title{
Dynamic Resource Allocation for Full-Duplex OFDMA Wireless Cellular Networks
}

\author{
Tam Thanh Tran, Vu Nguyen Ha, Long Bao Le, and André Girard \\ INRS, University of Quebec, Montréal, Québec, Canada. \\ E-mails: \{tam.tran,hanguyen,long.le\}@emt.inrs.ca, andre.girard@gerad.ca.
}

\begin{abstract}
This paper focuses on the resource allocation in a full-duplex (FD) multiuser single cell system consisting of one FD base-station (BS) and multiple FD mobile nodes. In particular, we are interested in jointly optimizing the power allocation (PA) and subcarrier assignment (SA) for uplink (UL) and downlink (DL) transmission of all users to maximize the system sumrate. First, the joint optimization problem is formulated as nonconvex mixed integer program, a difficult nonconvex problem. We then propose an iterative algorithm to solve this problem. In the proposed algorithm, the $\mathrm{PA}$ is obtained by employing the SCALE algorithm, whereas the SA is updated by a gradient method. Finally, we present numerical results to demonstrate the significant gains of our proposed design compared to that due to two fast greedy algorithms.
\end{abstract}

\section{INTRODUCTION}

Full-duplex (FD) has recently been considered as a technology evolution for the next-generation wireless network expecting significant capacity increase $[1,2]$. In FD systems, a wireless terminal can transmit and receive signal on the same frequency band at a particular time slot. Therefore, FD systems can potentially double the spectral efficiency relatively to the Half-duplex (HD) ones [1, 2]. The advantages of FD have come from the combination of various techniques, including antenna design, analog cancellation, and digital cancellation, to effectively reduces self-interference caused by the transmitted signal of a terminal to itself [3].

Nevertheless, the performance of the FD sytem cannot be as good since it still depends on the resource allocation design [4]. There have been many studies focusing on this topic. In [5], a power control protocol has been studied to maximize the minimum signal-to-interference-plus-noise (SINR) of all UL and DL links. In [6], Ju et al. jointly optimizes the time and power allocations in the wireless-power communication network to maximize the user's weighted sum rate. However, in these works, it is assumed that the FD technique is employed only at AP, where the users still work in HD mode. Additionally, the SA is not considered. Recently, [7] studies on jointly optimizing PA and SA design for the wireless network consisting FD base station and FD users. However, perfect SI cancellation is considered in this work where SI is treated as noise, which is not practical.

In this paper, we study a single cell OFDMA network where FD mode is enabled at all terminals (base station and users). Our objective is to maximize the total rate of the network by jointly optimizing SA and PA. Different to that modelled in [7], the SI in our paper is modelled generally as monomial form of transmission power (eq. (8) in [8]).
First, our considered joint optimization problem is formulated as nonconvex mixed integer program, which is NP-hard in general. Hence, an iterative algorithm is developed to solve this problem efficiently. In the proposed algorithm, PA is obtained by employing the SCALE algorithm [9], whereas the SA is updated by a gradient method [10]. Then, two greedy algorithms will be further introduced to demonstrate the significant gains of our proposed design.

\section{System Model AND Problem Formulation}

\section{A. System Model}

We consider a single cell OFDMA network consisting one base station (BS) and $N$ mobile nodes with FD transmission strategy. In particular, BS and every node are assumed to be able to transmit and receive signals simultaneously on any subcarrier (SC). The entire frequency band is partitioned into $\mathcal{S}$ orthogonal SCs. Let $\mathcal{N}=\{1,2, \ldots, N\}$ and $\mathcal{S}=\{1,2, \ldots, S\}$ denote the set of nodes and the set of SCs, respectively. We assume that each SC is assigned to at most one user for UL transmission and also at most one user for DL transmission. We denote a SA pattern for the UL and DL sides by binary vectors $\mathbf{X}^{\mathrm{U}} \triangleq\left\{x_{n, s}^{\mathrm{U}}\right\}_{n \in \mathcal{N}, s \in \mathcal{S}}$ and $\mathbf{X}^{\mathrm{D}} \triangleq\left\{x_{n, s}^{\mathrm{D}}\right\}_{n \in \mathcal{N}, s \in \mathcal{S}}$, respectively, where element $x_{n, s}^{\mathrm{U}}$ and $x_{n, s}^{\mathrm{U}}$ 's are defined as

$$
x_{n, s}^{\mathrm{U}}\left(\text { or } x_{n, s}^{\mathrm{D}}\right)=\left\{\begin{array}{l}
1 \text { if user } n \text { uses SC } s \text { for UL (or DL), } \\
0 \text { otherwise. }
\end{array}\right.
$$

Let $p_{n, s}^{\mathrm{U}}$ denote the UL power allocated to SC $s$ by node $n$ and $p_{n, s}^{\mathrm{D}}$ denote the DL power allocated to SC $s$ for node $n$ by BS. We define PA vector $\mathbf{P}$ as $\mathbf{P} \triangleq\left\{\mathbf{p}_{s}^{\mathrm{U}}, \mathbf{p}_{s}^{\mathrm{D}}\right\}_{s \in \mathcal{S}}$ where $\mathbf{p}_{s}^{\mathrm{U}}$ and $\mathbf{p}_{s}^{\mathrm{D}}$ are the upstream and downstream power vectors for SC $s$, respectively. BS is assumed to know the perfect channel information for each node and SC. We are now ready for investigating the rates achieved by all nodes based on which we can define our problem.

1) Rate achieved at the base station: Due to the FD transmission strategy, the received signal at BS over a SC is interfered by the transmitted signal from BS over that SC. Although interference cancellation is employed to reduce this interference, it is imperfect. Hence, there exists residual selfinterference in each SC. According to [8], the self-interference on UL from DL transmission corresponding to SC $s$ can be given as

$$
I_{s}^{\mathrm{U}}\left(\mathbf{x}_{s}^{\mathrm{D}}, \mathbf{p}_{s}^{\mathrm{D}}\right)=\sum_{n \in \mathcal{N}} x_{n, s}^{\mathrm{D}} \epsilon\left(p_{n, s}^{\mathrm{D}}\right)^{\theta},
$$


where $\mathbf{x}_{s}^{\mathrm{D}}=\left\{x_{n, s}^{\mathrm{D}}\right\}_{n \in \mathcal{N}}$ is the DL SA vector for SC $s$, $\epsilon$ and $\theta$ are fixed scaling factors which reflect the quality of the selected cancellation techniques. In the worst case, the self-interference power can be well approximated as a linear function of the transmitted power [6], i.e $\theta=1$. In the optimistic case, $\theta=0$, the self-interference is a constant regardless of the transmission power [7]. Then, the rate achieved at the base station for the user $u$ on the UL transmission over SC $s$ can be described as

$$
R_{n, s}^{\cup}(\mathbf{X}, \mathbf{P})=\log \left(1+\frac{x_{n, s}^{\mathrm{U}} G_{n, s}^{\mathrm{U}} p_{n, s}^{\mathrm{U}}}{I_{s}^{\mathrm{U}}\left(\mathbf{x}_{s}^{\mathrm{D}}, \mathbf{p}_{s}^{\mathrm{D}}\right)+N_{0}}\right),
$$

where $G_{n, s}^{U}$ is UL channel gain between BS and node $n$, and $N_{0}$ is the receiver noise floor.

2) Rate achieved at the users: Let assume that the user $n$ is assigned SC $s$ over DL. Then, there are two cases which can happen. The first one is that the same SC $s$ is also assigned to user $n$ for UL transmission. In this case, the self-interference occurs and this interference can be given as $\epsilon\left(p_{n, s}^{U}\right)^{\theta}$. The second case is that $\mathrm{SC} s$ is assigned to another user, i.e., user $j$, for UL transmission. Then, the interference in this case is cross-interference caused by the UL transmission from user $j$ on the received DL signal user $n$ over $\mathrm{SC} s$, and this interference can be calculated as

$$
I_{j, n, s}^{\mathrm{cr}}\left(\mathbf{x}_{s}^{\mathrm{U}}, \mathbf{p}_{s}^{\mathrm{U}}\right)=x_{j, s}^{\mathrm{U}} G_{j, n, s} p_{j, s}^{\mathrm{U}},
$$

where $G_{n, j, s}$ is the interference channel gain from user $n$ to user $j$ over SC $s$ and $\mathbf{x}_{s}^{\mathrm{U}}=\left\{x_{n, s}^{\mathrm{U}}\right\}_{n \in \mathcal{N}}$ is UL SA vector for $\mathrm{SC} s$. Generally, the interference on the received signal of user $n$ over SC $s$ can be summarized as follows.

$$
I_{n, s}^{\mathrm{D}}\left(\mathbf{x}_{s}^{\mathrm{U}}, \mathbf{p}_{s}^{\mathrm{U}}\right)=\sum_{j \in \mathcal{N} / n} x_{j, s}^{\mathrm{U}} G_{j, n, s} p_{j, s}^{\mathrm{U}}+x_{n, s}^{\mathrm{U}} \epsilon\left(p_{n, s}^{\mathrm{U}}\right)^{\theta} .
$$

Then, the rate achieved by the user $u$ on the DL transmission over SC $s$ can be described as

$$
R_{n, s}^{\mathrm{D}}(\mathbf{X}, \mathbf{P})=\log \left(1+\frac{x_{n, s}^{\mathrm{D}} G_{n, s}^{\mathrm{D}} p_{n, s}^{\mathrm{D}}}{I_{n, s}^{\mathrm{D}}\left(\mathbf{x}_{s}^{\mathrm{U}}, \mathbf{p}_{s}^{\mathrm{U}}\right)+N_{0}}\right),
$$

where $G_{n, s}^{\mathrm{D}}$ is DL channel gain between BS and node $n$.

\section{B. Problem Formulation}

In this following, we formulate for the joint FD SA and PA problem. This problem aims to determine the SA for users in DL ans UL transmission separately as well as the corresponding power transmission for each link to maximize the total rate of the network. Due to these objectives, our problem can be stated as

$$
\begin{aligned}
\max _{\mathbf{X}, \mathbf{P}} & R_{\text {total }}=\sum_{n \in \mathcal{N}} \sum_{s \in \mathcal{S}} R_{n, s}^{\mathrm{U}}(\mathbf{X}, \mathbf{P})+R_{n, s}^{\mathrm{D}}(\mathbf{X}, \mathbf{P}) \\
\text { s.t. } & \sum_{n \in \mathcal{N}} \sum_{s \in \mathcal{S}} p_{n, s}^{\mathrm{D}} \leq P_{\mathrm{BS}}, \\
& \sum_{s \in \mathcal{S}} p_{n, s}^{\mathrm{U}} \leq P_{n}, \forall n \in \mathcal{N}, \\
& \sum_{n \in \mathcal{N}} x_{n, s}^{\mathrm{U}} \leq 1 \text { and } \sum_{n \in \mathcal{N}} x_{n, s}^{\mathrm{D}} \leq 1, \forall s \in \mathcal{S}, \\
& x_{n, s}^{\mathrm{U}}, x_{n, s}^{\mathrm{D}} \in\{0,1\}, \forall n \in \mathcal{N}, \forall s \in \mathcal{S} .
\end{aligned}
$$

In the above optimization problem, $P_{\mathrm{BS}}$ and $P_{n}$ denote the maximum transmission power budget at $\mathrm{BS}$ and user $n$, respectively. The constraints (7d) impose the assignment of $\mathrm{SC} s$ to only one user for UL or DL transmissions. It is further observed that the optimization problem (7) is indeed a mixed integer program, which is NP-hard in general and requires exponential complexity to be solved. Inspired by a recent work in PA and SA [9], an iterative algorithm is developed in the subsequent sections to obtain locally optimal solution to problem (7).

\section{Sum-Rate Maximization with Given SA}

\section{A. Reformulation}

In this section, we first reformulate the problem (7) into a sum-rate maximization problem when $x_{n, s}^{\mathrm{U}}$ 's $x_{n, s}^{\mathrm{U}}$ 's are known. Then a numerical method is investigated to solve this problem. For simplicity, we use new power variables representing the transmission power for all users on each SC. In particular, let us denote $q_{s}^{\mathrm{U}}$ and $q_{s}^{\mathrm{D}}$ as the transmission power over SC $s$ on the UL side and DL side, respectively. The relation between these power variables and the power of BS and users on SCs can be given as

$$
q_{s}^{\mathrm{U}}=\sum_{n \in \mathcal{N}} x_{n, s}^{\mathrm{U}} p_{n, s}^{\mathrm{U}}, \quad q_{s}^{\mathrm{D}}=\sum_{n \in \mathcal{N}} x_{n, s}^{\mathrm{D}} p_{n, s}^{\mathrm{D}} .
$$

Then, the self-interference over SC $s$ on UL transmission can be rewritten as $I_{s}^{\prime} \mathrm{U}\left(q_{s}^{\mathrm{D}}\right)=\epsilon\left(q_{s}^{\mathrm{D}}\right)^{\theta}$. Based on this result, the rate for UL transmission over SC $s$ can be calculated as

$$
R_{s}^{\prime}{ }^{\mathrm{U}}\left(\mathbf{X}, q_{s}^{\mathrm{D}}, q_{s}^{\mathrm{U}}\right)=\log \left(1+\frac{q_{s}^{\mathrm{U}} G_{s}^{\mathrm{U}}(\mathbf{X})}{\epsilon\left(q_{s}^{\mathrm{D}}\right)^{\theta}+N_{0}}\right),
$$

where $G_{s}^{\mathrm{U}}(\mathbf{X})=\sum_{n \in \mathcal{N}} x_{n, s}^{\mathrm{U}} G_{n, s}^{\mathrm{U}}$. On the DL side, the interference over SC $s$ can be given as

$$
I_{s}^{\prime} \mathrm{D}\left(\mathbf{X}, q_{s}^{\mathrm{U}}\right)=q_{s}^{\mathrm{U}} G_{s}^{\mathrm{C}}(\mathbf{X})+\epsilon_{s}^{\mathrm{U}}(\mathbf{X})\left(q_{s}^{\mathrm{U}}\right)^{\theta},
$$

where $G_{s}^{\mathrm{C}}(\mathbf{X})=\sum_{n \in \mathcal{N}} \sum_{j \in \mathcal{N} / n} x_{n, s}^{\mathrm{D}} x_{j, s}^{\mathrm{U}} G_{j, n, s}$ and $\epsilon_{s}^{\mathrm{U}}(\mathbf{X})=$ $\epsilon \sum_{n \in \mathcal{N}} x_{n, s}^{\mathrm{D}} x_{n, s}^{\mathrm{U}}$. Then, the rate for DL transmission over SC $s$ can be calculated as

$$
R_{s}^{\prime \mathrm{D}}\left(\mathbf{X}, q_{s}^{\mathrm{D}}, q_{s}^{\mathrm{U}}\right)=\log \left(1+\frac{q_{s}^{\mathrm{D}} G_{s}^{\mathrm{D}}(\mathbf{X})}{I_{s}^{\prime} \mathrm{D}\left(\mathbf{X}, q_{s}^{\mathrm{U}}\right)+N_{0}}\right),
$$

where $G_{s}^{\mathrm{D}}(\mathbf{X})=\sum_{n \in \mathcal{N}} x_{n, s}^{\mathrm{D}} G_{n, s}^{\mathrm{D}}$. Let $\mathbf{Q} \triangleq\left\{q_{s}^{\mathrm{U}}, q_{s}^{\mathrm{D}}\right\}_{s \in \mathcal{S}}$. Then, the problem (7) is equivalent to the sum-rate maximization problem when the SA is given

$$
\begin{aligned}
\max _{\mathbf{Q}} & R_{\mathbf{X}}(\mathbf{Q})=\sum_{s \in \mathcal{S}}\left[\log \left(1+\frac{G_{s}^{\mathrm{U}}(\mathbf{X}) q_{s}^{\mathrm{U}}}{\epsilon\left(q_{s}^{\mathrm{D}}\right)^{\theta}+N_{0}}\right)\right. \\
+ & \left.\log \left(1+\frac{G_{s}^{\mathrm{D}}(\mathbf{X}) q_{s}^{\mathrm{D}}}{G_{s}^{\mathrm{C}}(\mathbf{X}) q_{s}^{\mathrm{U}}+\epsilon_{s}^{\mathrm{U}}(\mathbf{X})\left(q_{s}^{\mathrm{U}}\right)^{\theta}+N_{0}}\right)\right] \\
\text { s.t. } & \sum_{s \in \mathcal{S}} q_{s}^{\mathrm{D}} \leq P_{\mathrm{BS}}, \\
& \sum_{s \in \mathcal{S}} x_{n, s}^{\mathrm{U}} q_{s}^{\mathrm{U}} \leq P_{n}, \forall n \in \mathcal{N} .
\end{aligned}
$$


In the above optimization problem, the objective function is non-convex. Hence, it is very difficult to find the optimal solution of this problem. In the next section, we leverage the SCALE algorithm in [9] to solve (11).

\section{B. SCALE-Based Algorithm}

In this section, we apply the SCALE algorithm [9] to find the PA for each user on each transmission side and each SC when the SA is assumed given. The idea of this numerical method is to relax the non-convex objective function to its lower bound which is convex with respect to $q_{s}^{\mathrm{U}}$ 's and $q_{s}^{\mathrm{D}}$ 's in order to obtain a relaxed convex problem. This convex problem is then solved optimally. Then, the lower bound is updated iteratively to tighten the non-convex objective function at the optimal point.

We make use of the following lower bound, for any $z \geq 0$ and $\bar{z} \geq 0$ as in [9] as

$$
\log (1+z) \geq \alpha_{\bar{z}} \log z+\beta_{\bar{z}},
$$

that is tight at $z=\bar{z}$, where

$$
\alpha_{\bar{z}}=\frac{\bar{z}}{1+\bar{z}}, \beta_{\bar{z}}=\log (1+\bar{z})-\frac{\bar{z}}{1+\bar{z}} \log \bar{z} .
$$

We use the convention that $\log (0)=-\infty$ and $0 \log (0)=0$. By using (12), we have

$$
\begin{aligned}
& R_{\mathbf{X}}(\mathbf{Q}) \geq \bar{R}_{\mathbf{X}}(\mathbf{Q}, \alpha, \beta) \triangleq \sum_{s=1}^{S}\left[\alpha_{s}^{\mathrm{U}} \log \left(\frac{G_{s}^{\mathrm{U}}(\mathbf{X}) q_{s}^{\mathrm{U}}}{\epsilon\left(q_{s}^{\mathrm{D}}\right)^{\theta}+N_{0}}\right)+\beta_{s}^{\mathrm{U}}\right. \\
& \left.+\alpha_{s}^{\mathrm{D}} \log \left(\frac{G_{s}^{\mathrm{D}}(\mathbf{X}) q_{s}^{\mathrm{D}}}{G_{s}^{\mathrm{C}}(\mathbf{X}) q_{s}^{\mathrm{U}}+\epsilon_{s}^{\mathrm{U}}(\mathbf{X})\left(q_{s}^{\mathrm{U}}\right)^{\theta}+N_{0}}\right)+\beta_{s}^{\mathrm{D}}\right] .
\end{aligned}
$$

We denote $\alpha \triangleq\left\{\alpha_{s}^{\mathrm{U}}, \alpha_{s}^{\mathrm{D}}\right\}$ and $\beta \triangleq\left\{\beta_{s}^{\mathrm{U}}, \beta_{s}^{\mathrm{D}}\right\}$. The terms $\alpha_{s}^{\mathrm{U}}$ and $\beta_{s}^{\mathrm{U}}$ are corresponding to the relaxation of UL and DL part in $R_{s}^{U}$. Similarly, the terms $\alpha_{s}^{\mathrm{D}}$ and $\beta_{s}^{\mathrm{D}}$ are corresponding to the relaxation of UL and DL part in $R_{s}^{\mathrm{D}}$. It can be noted that the bound (14) is tight at

$$
\begin{aligned}
& \bar{z}_{s}^{\mathrm{U}}=\Gamma_{s, \mathbf{X}}^{\mathrm{U}}(\mathbf{Q})=\frac{G_{s}^{\mathrm{U}}(\mathbf{X}) q_{s}^{\mathrm{U}}}{\epsilon\left(q_{s}^{\mathrm{D}}\right)^{\theta}+N_{0}}, \\
& \bar{z}_{s}^{\mathrm{D}}=\Gamma_{s, \mathbf{X}}^{\mathrm{D}}(\mathbf{Q})=\frac{G_{s}^{\mathrm{D}}(\mathbf{X}) q_{s}^{\mathrm{D}}}{G_{s}^{\mathrm{C}}(\mathbf{X}) q_{s}^{\mathrm{U}}+\epsilon_{s}^{\mathrm{U}}(\mathbf{X})\left(q_{s}^{\mathrm{U}}\right)^{\theta}+N_{0}} .
\end{aligned}
$$

By using transformation $\tilde{\mathbf{Q}}=\log \mathbf{Q}$, we obtain

$$
\begin{aligned}
& \bar{R}_{\mathbf{X}}(\tilde{\mathbf{Q}}, \alpha, \beta)=\sum_{s=1}^{S}\left[\alpha_{s}^{\mathrm{U}} \tilde{q}_{s}^{\mathrm{U}}+\eta_{s}^{\mathrm{U}}-\alpha_{s}^{\mathrm{U}} \log \left(\epsilon e^{\theta \tilde{q}_{s}^{\mathrm{D}}}+N_{0}\right)\right. \\
& \left.+\alpha_{s}^{\mathrm{D}} \tilde{q}_{s}^{\mathrm{D}}+\eta_{s}^{\mathrm{D}}-\alpha_{s}^{\mathrm{D}} \log \left(G_{s}^{\mathrm{C}}(\mathbf{X}) e^{\tilde{q}_{s}^{\mathrm{D}}}+\epsilon_{s}^{\mathrm{U}}(\mathbf{X}) e^{\theta \tilde{q}_{s}^{\mathrm{U}}}+N_{0}\right)\right]
\end{aligned}
$$

where $\eta_{s}^{\mathrm{U}}=\alpha_{s}^{\mathrm{U}} \log G_{s}^{\mathrm{U}}(\mathbf{X})+\beta_{s}^{\mathrm{U}}$ and $\eta_{s}^{\mathrm{D}}=\alpha_{s}^{\mathrm{D}} \log G_{s}^{\mathrm{D}}(\mathbf{X})+$ $\beta_{s}^{\mathrm{D}}$. As can be observed, $\bar{R}_{\mathbf{X}}(\tilde{\mathbf{Q}}, \alpha, \beta)$ is a concave function of $\tilde{\mathbf{Q}}$. Then, for given $\alpha, \beta$, the relaxation of problem (11) can be described as follows:

$$
\begin{aligned}
\max _{\tilde{\mathbf{Q}}} & \bar{R}_{\mathbf{X}}(\tilde{\mathbf{Q}}, \alpha, \beta) \\
\text { s.t. } & \sum_{s \in \mathcal{S}} e^{\tilde{q}_{s}^{\mathrm{D}} \leq P_{\mathrm{BS}},} \\
& \sum_{s \in \mathcal{S}} x_{n, s}^{\mathrm{U}} e^{\tilde{q}_{s}^{U}} \leq P_{n}, \forall n \in \mathcal{N},
\end{aligned}
$$

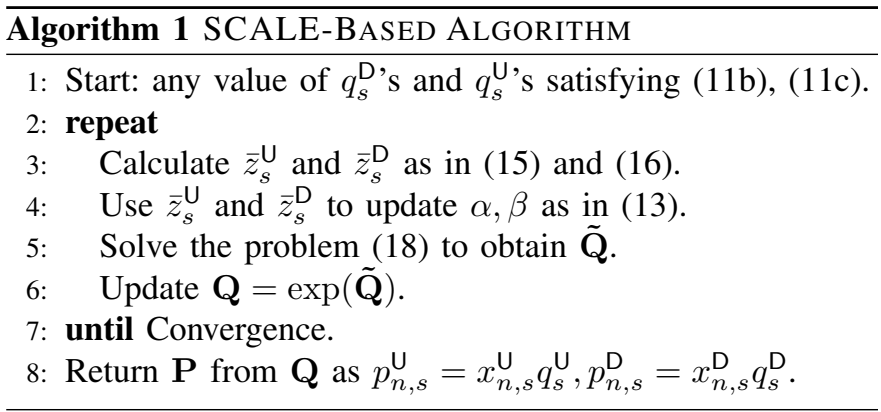

We now have a standard convex maximization problem that is efficiently solved in the dual domain. Once a solution is obtained, we may transform back to $\mathrm{Q}$-space with $\mathbf{Q}=\exp (\tilde{\mathbf{Q}})$. Here we are optimizing the lower bound of $R_{\mathbf{X}}(\mathbf{Q})$. As a result, it is natural to tighten the bound successively by updating the choice of $\alpha, \beta$ according to the new power transmission solution. Then, by iteratively updating $\bar{z}_{s}^{\mathrm{U}}$ 's, $\bar{z}_{s}^{\mathrm{D}}$ 's as well as $\alpha, \beta$, we obtain the SCALE-Based algorithm to maximize DL and UL sum-rate, as summarized in Algorithm 1. The convergence of the algorithm is guaranteed due to the following Proposition.

Proposition 1. The Algorithm 1 converges after a finite number of iterations.

Proof. By iteratively updating $\bar{z}_{s}^{\mathrm{U}}$ 's, $\bar{z}_{s}^{\mathrm{D}}$ 's, and $\alpha, \beta$ as in (15), (16), and (13), we always have the fact that the optimal solution of problem (18) in the previous iteration is a feasible point in the feasible set of that problem in the next iteration. Hence, the convergence of the algorithm is guaranteed due to the monotonic increase of the objective function in problem (18) after each iteration. Hence, the Algorithm 1 will converge after a finite number of iterations.

\section{SA Problem With Given PA}

This section investigates the next step in finding a good SA for all users with given PA over all SCs for downstream and upstream. Assume $\mathbf{Q}$ is given, we replace the quadratic terms $x_{n, s}^{\mathrm{D}} x_{j, s}^{\mathrm{U}}$ in $\epsilon_{s}^{\mathrm{U}}(\mathbf{X})$ by the following binary variables

$$
y_{n, j, s}=x_{n, s}^{\mathrm{D}} x_{j, s}^{\mathrm{U}}, \quad \forall n, j \in \mathcal{N} .
$$

As can be observed, $y_{n, j, s}=1$ if $\mathrm{SC} s$ is assigned to user $n$ in DL and to user $j$ in UL, and $y_{n, j, s}=0$, otherwise. When $y_{n, j, s}=1$, the rate achieved over SC $u$ for given $\mathbf{Q}$ can be calculated as

$$
\begin{aligned}
R_{n, j, s}= & \log \left(1+\frac{G_{j, s}^{\mathrm{U}} q_{s}^{\mathrm{U}}}{\epsilon\left(q_{s}^{\mathrm{D}}\right)^{\theta}+N_{0}}\right) \\
& +\log \left(1+\frac{G_{n, s}^{\mathrm{D}} q_{s}^{\mathrm{D}}}{I_{\mathbf{Q}, s}^{\prime}(n, j)+N_{0}}\right),
\end{aligned}
$$

where

$$
I_{\mathbf{Q}, s}^{\prime}(n, j)= \begin{cases}\epsilon\left(q_{s}^{\mathrm{U}}\right)^{\theta} & \text { if } n=j \\ G_{j, n, s} q_{s}^{\mathrm{U}} & \text { if } n \neq j .\end{cases}
$$


Given a value of $q_{s}^{U}$ and $q_{s}^{D}$, we optimize the allocation $y_{n, j, s}$ :

$$
\begin{aligned}
\max _{\mathbf{Y}} & R_{\mathbf{Q}}(\mathbf{Y})=\sum_{\forall(n, j)} \sum_{s \in \mathcal{S}} R_{n, j, s} y_{n, j, s} \\
\text { s.t. } & \sum_{n \in \mathcal{N}} \sum_{s \in \mathcal{S}} q_{s}^{\cup} y_{n, j, s} \leq P_{j}, \quad \forall j \in \mathcal{N}, \\
& \sum_{\forall(n, j)} y_{n, j, s} \leq 1, \quad s \in \mathcal{S}, \\
& y_{n, j, s} \in\{0,1\}, \forall(n, j), \forall s \in \mathcal{S},
\end{aligned}
$$

where $\mathbf{Y}$ represents the vector generated from all variables $y_{n, j, s}$ 's. While problem (22) is a nonconvex integer program, its globally optimization can be found. By relaxing the last constraint (22d) into $0 \leq y_{n, j, s} \leq 1$, the problem (22) becomes a linear program (LP) in $y_{n, j, s}$ 's, whose optimal solution can be found efficiently by standard convex optimization techniques. Interesting, as shown in [10] the obtained optimal solution of this LP lies at a vertex where $y_{n, j, s}$ 's will be either 0 and 1 . Thus, given $\mathbf{Q}$, as well as $R_{n, j, s}$ 's, solving the LP will result in the optimal SA for each user. However, once $y_{n, j, s}$ 's take the value of 0 or 1 , the SA for each user may be trapped in a local optimal point based on which the PA cannot be optimized anymore. To overcome this critical issue, the gradient method can be adopted to "slowly" update the variable $y_{n, j, s}$ 's as in [10]. Once, $y_{n, j, s}$ 's are changed slowly, the PA can be optimized more carefully; then, the better solution hence can be achieved [10]. In particular, the gradient of $y_{n, j, s}$ as in objective function of problem (22) can be given as $R_{n, j, s}$. Then, the gradient projection method can be described as follows:

$$
\mathbf{Y}^{\mathbf{k}+\mathbf{1}}=P_{\Omega}\left(\mathbf{Y}^{\mathbf{k}}+\lambda^{k} \mathbf{R}\right)
$$

where $\mathbf{R}$ represents the vector generated from $R_{n, j, s}$ 's, $\lambda_{k}$ is the a small diminishing gradient step size, and $P_{\Omega}(\cdot)$ denotes the projection which is further described as following quadratic problem.

$$
\begin{array}{ll}
\min _{\mathbf{Y}} & \left\|y_{n, j, s}-\hat{y}_{n, j, s}\right\|^{2} \\
\text { s.t. } & \text { constraints }(22 \mathrm{~b}) \text { and }(22 \mathrm{c}), \\
& 0 \leq y_{n, j, s} \leq 1, \forall(n, j), \forall s \in \mathcal{S},
\end{array}
$$

where $\hat{y}_{n, j, s}=y_{n, j, s}^{k}+\lambda^{k} R_{n, j, s}$. The above updating process can guarantee to converge to the local optimal value of $y_{n, j, s}$ 's if the $\lambda_{k}$ is chosen carefully so that $\lambda_{k} \rightarrow 0$ when $k \rightarrow \infty$ [10]. We summarize the combination of update $y_{n, j, s}$ 's and Algorithm 1 in Algorithm 2 which can be applied to solve the problem (7).

Proposition 2. The iterative procedure presented in Algorithm 2 converges after a finite number of iterations.

Proof. Through Proposition 1, the objective function of problem (18) increases after each iteration. Hence, the sum-rate will be improved by Algorithm 1. In addition, the gradient update of $y_{n, j, s}$ 's further improves this sum-rate value. Therefore, Algorithm 2 will monotonically increase the objective function of problem (7), which leads to the convergence.

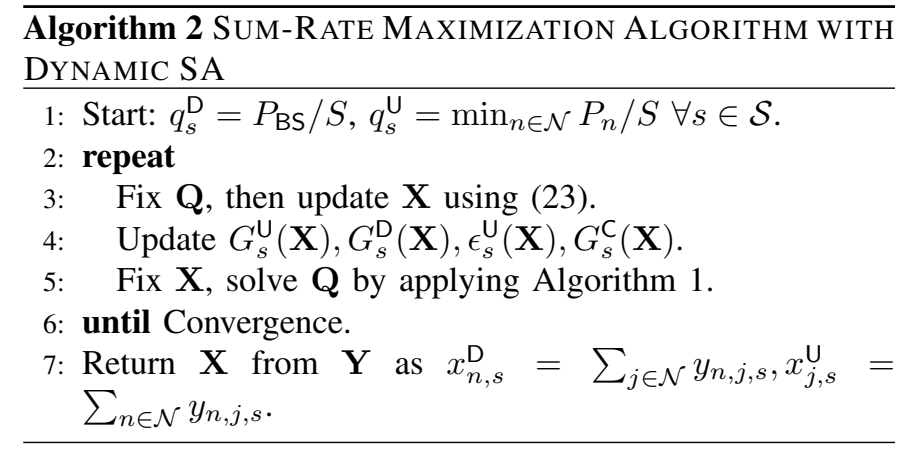

\section{GREedy Algorithms}

For comparison purposes, we introduce two fast greedy algorithms in this section, which are designed for FD mode and HD mode, respectively.

1) Full-Duplex Greedy Algorithm: In this algorithm, we start with a uniform PA for each DL communication. For the UL side, since the number of SCs assigned to a node is needed for allocating the power, the greedy algorithm is employed as follows:

a. For each SC in $\mathcal{S}$, we compute the rate (20) for each pair of $(n, j)$. Assuming that SC $s$ is assigned to node $j$, then the UL power of $s$ can be determined in order to calculate the rate.

b. Let $\left(n^{*}, j^{*}\right)$ be the pair of $(n, j)$ which offers the highest rate when $s$ is assigned. Then SC $s$ will be allocated to $\left(n^{*}, j^{*}\right)$.

c. Once all of SCs in $\mathcal{S}$ have been assigned, we return the SA result $\mathbf{X}$ and update the uniform PA $\mathbf{P}$.

2) Half-Duplex Greedy Algorithm: In this algorithm, each $\mathrm{SC}$ is assigned for only one side transmission, UL or DL. This algorithm is designed similar to the FD greedy algorithm. First, the uniform power distribution is employed for this method as well. Then, for SC allocation, each SC is assigned for the user with its DL or UL transmission which correspond the highest achievable rate in stead of a pair of UL and DL transmission as in the FD algorithm.

\section{Simulation Results}

We consider a single cell network in which the users are allocated randomly so that the distances from them to BS are in the range between $50 \mathrm{~m}$ and $100 \mathrm{~m}$. The channel gains are generated by considering both Rayleigh fading and path loss which is modelled based on the WINNER-II Model as $P L(d)=A \log _{10}(d)+B+C \log _{10}\left(f_{c} / 5\right)$, where $A=36.8$, $B=43.8, C=23, f_{c}=2.5 G H z$, and $d$ is the distance between two terminals. We also choose the number of users as 10 and that of SCs as 20 except Fig. 4. The power at BS and users are set as $4 W$ and $0.4 W$, respectively. In Fig. 3, these power are scaled with a scaling factor $\Omega$. The noise power is chosen as $N_{0}=10^{-12} \mathrm{~W}$.

First, we examine the convergence of our proposed algorithms by showing the variations of system sum rate corresponding to different values of $\epsilon$ over iterations by using the combination of Algorithm 1 and Algorithm 2 ("Proposed 


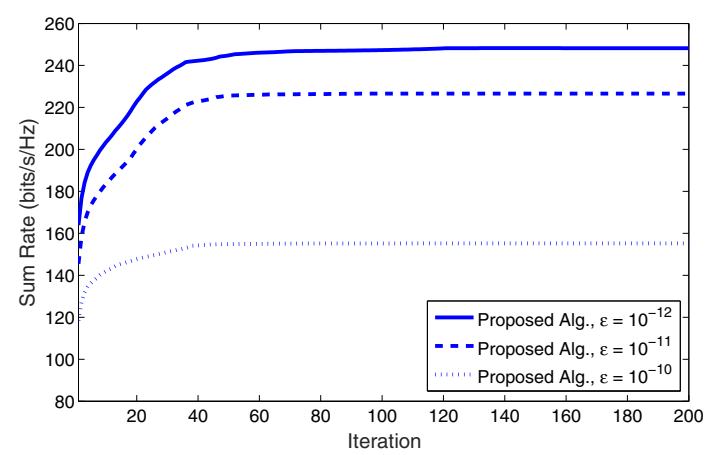

Fig. 1. Total rate versus the iteration index.

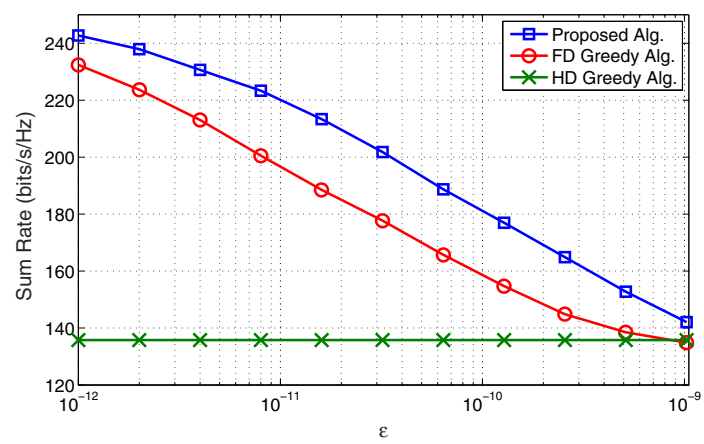

Fig. 2. Total rate versus the interference factor $\epsilon$.

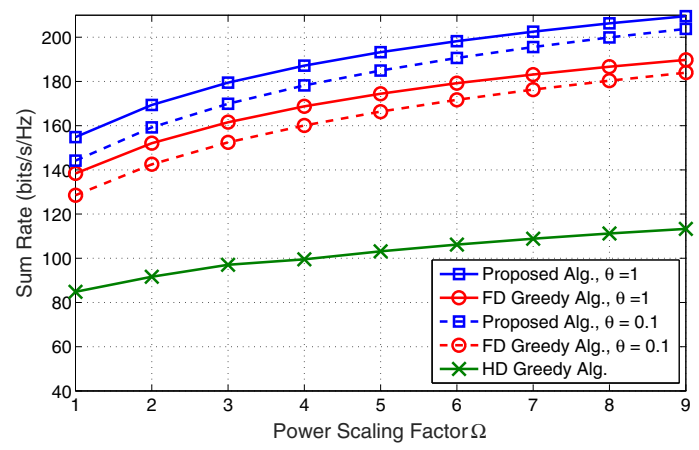

Fig. 3. Total rate versus the power transmission.

Alg.") in Fig. 1. To obtain these simulation results, we set $\theta=1$. It can be observed that our proposed algorithm converge for both inner and outer loops.

In Figs. 2-4, we present the variations of system sum rate obtained by our proposed algorithm and two greedy algorithms - "FD Greedy Alg." and "HD Greedy Alg." over the changes of some network parameters, e.g., the interference factor $\epsilon$ in Fig. 2, the power transmission in Fig. 3, and the number of SCs in Fig. 4. As can been observed, our proposed algorithm outperforms two greedy algorithms in all studied scenarios. Moreover, the FD technique shows its advantage by achieving a higher rate than the HD mode does.

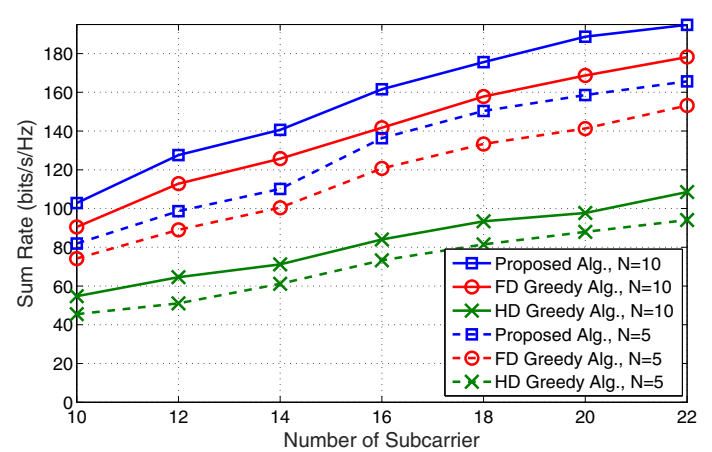

Fig. 4. Total rate versus the number of subcarriers.

Interestingly, Fig. 2 illustrates that higher value of $\epsilon$ results in the lower system sum rate of the FD mode ("Proposed Alg." and "FD Greedy Alg."). This can be explained by the fact that the higher value of $\epsilon$ is equivalent to the worse SI cancellation which reduces the benefits of FD technique. Fig. 3 shows that increasing the transmission power of terminals results in the better sum rate achieved by the network as expected. In addition, the higher number of SCs also leads to the higher network sum rate as illustrated in Fig. 4.

\section{CONCLUSION}

We have proposed a novel algorithm for joint PA and SA design in FD single cell network which aims to maximize the system sum rate subject to constraints on the power transmission limit. Numerical results have illustrated the efficacy of our proposed algorithms and the impacts of different parameters on the sum-rate performance.

\section{REFERENCES}

[1] D. Kim, H. Lee, and D. Hong, "A survey of in-band full-duplex transmission: From the perspective of PHY and MAC layers," IEEE Commun. Surveys Tuts., vol. 17, no. 4, pp. 2017-2046, 4th Quarter 2015.

[2] M. Duarte and A. Sabharwal,"Full-duplex wireless communications using off-the-shelf radios: Feasibility and first results," in Proc. of IEEE ASILOMAR, 2010.

[3] E. Everett, A. Sahai, and A. Sabharwal, "Passive self-interference suppression for full-duplex infrastructure nodes," IEEE Trans. Wireless Commun., vol. 13, no. 2, pp. 680-694, Feb. 2014.

[4] L. Song, Y. Li, and Z. Han, "Resource allocation in full-duplex communications for future wireless networks," IEEE Wireless Commun., vol. 22, no. 4, pp. 88-96, Aug. 2015.

[5] W. Choi, H. Lim, and A. Sabharwal, "Power-controlled medium access control protocol for full-duplex wifi networks," IEEE Trans. Wireless Commun., vol. 14, no. 7, pp. 3601-3613, May 2015.

[6] H. Ju and R. Zhang, "Optimal resource allocation in full-duplex wirelesspowered communication network," IEEE Trans. Commun., vol. 62, no. 10, pp. 3528-3540, Oct. 2014.

[7] C. Nam, C. Joo and S. Bahk, "Joint subcarrier assignment and power allocation in full-duplex OFDMA networks," IEEE Trans. Wireless Commun., vol. 14, no. 6, pp. 3108-3119, Jun. 2015.

[8] L. Jiménez Rodríguez, N. H. Tran, and T. Le-Ngoc, "Performance of full-duplex AF relaying in the presence of residual self-interference," IEEE J. Select. Areas in Commun., vol. 32, no. 9, pp. 1752-1764, Sept. 2014.

[9] L. Venturino, N. Prasad, and X. Wang, "Coordinated scheduling and power allocation in downlink multicell OFDMA networks," IEEE Trans. Veh. Technol., vol. 58, no. 6, pp. 2835-2848, Jul. 2009.

[10] M. Sanjabi, M. Razaviyayn, and Zhi-Quan Luo, "A unified convergence analysis of block successive minimization methods for nonsmooth optimization," SIAM J. Opt., , vol. 23, no. 2, pp. 1126-1153, 2013. 